\title{
$\mathrm{M}|\mathrm{R}| \mathrm{S}$ Internet Journal Nitride Semiconductor Research
}

\section{Modeling of InGaN MOVPE in AIX 200 Reactor and AIX 2000 HT Planetary Reactor}

\author{
R. A. Talalaev ${ }^{1}$, E. V. Yakovlev², S. Yu. Karpov², Yu. N. Makarov³ , O. Schoen ${ }^{4}$, M. Heuken ${ }^{4}$, G. \\ Strauch $^{4}$ and Holger Juergensen ${ }^{4}$ \\ ${ }^{1}$ Ioffe Physical-Technical Institute, \\ ${ }^{2}$ Soft-Impact Ltd (St.Petersburg, Russia), \\ ${ }^{3}$ Lehrstuhl für Strömungsmechanik, University of Erlangen-Nürnberg, \\ ${ }^{4} A I X T R O N A G$,
}

(Received Tuesday, March 9, 1999; accepted Friday, May 28, 1999)

\begin{abstract}
Multiwafer Planetary Reactor is a promising system for large-scale production of heterostructures for LED's based on III-group nitrides. Analysis of chemical processes occurring in the reactor allows one to get insight into specific mechanisms governing growth of nitride based heterostructures. In the present paper results of modeling analysis of MOVPE of $\operatorname{In}_{\mathrm{x}} \mathrm{Ga}_{1-\mathrm{x}} \mathrm{N}$ layers in AIX-200 Reactor and AIX 2000 HT Planetary Reactor are reported. The model used for MOVPE process analysis accounts for gas flow, heat transfer, and multicomponent mass transport along with gas phase and surface chemical reactions. Results of the modeling analysis of In transport and incorporation into the solid phase are compared with experimental data. It is shown that the model predicts reasonably well the In incorporation during MOVPE of $\mathrm{InGaN}$ under $\mathrm{In} /(\mathrm{In}+\mathrm{Ga})$ ratio in the gas phase less than $20 \%$.
\end{abstract}

\section{Introduction}

MOVPE of group III-nitrides is a growth technology widely used to fabricate heterostructures for green, blue and ultraviolet light emitting and laser diodes [1]. Efficient production of the nitrides based devices requires good reproducibility and reliability of the growth process. Promising results in group III-nitrides growth are obtained using AIX-family MOVPE reactors [2], [3].

One of the key issues in manufacturing of the nitrides based heterostructures is control of In-composition in the $\mathrm{In}_{x} \mathrm{Ga}_{1-x} \mathrm{~N}$ epitaxial layers. Experimental studies ( [4], [5]) reveal the dependence of indium content in InGaN on growth temperature as well as on In/ $(\mathrm{In}+\mathrm{Ga})$ ratio in the gas phase. There was pointed out that the character of these dependencies was more complicated in comparison with the case of other III-V alloys when the indium incorporation into the growing layer is mainly determined by its content in the gas phase. The role of carrier gas in the InGaN deposition process was studied by Piner et al. [6]. The indium incorporation was found to be significantly influenced by the amount of hydrogen flowing into the reactor. The increased In incorporation at high growth rates was reported by Keller et al [5] and Sohmer et al [7].
There was also found that indium incorporation could be increased by increasing the gallium precursor flow only while all other process parameters are kept constant [8]. It should be noted that such a variation leads to lowering of indium gas phase composition $\mathrm{In} /(\mathrm{In}+\mathrm{Ga})$ and such behavior is very non-ordinary.

Optimization of indium incorporation into the growing epitaxial layer requires understanding of specific features of transport of In-containing species in the gas phase as well as mechanisms of their interactions with the surface. A detailed experimental investigation of these phenomena is complicated because of the number of techniques allowing in-situ diagnostics of the growth process is limited. In this case modeling analysis may provide additional insights into the underlying basic physical and chemical processes during MOVPE and may be a useful tool in the evaluation and interpretation of the role of different growth parameters affecting indium incorporation into the solid phase. Up to now only few modeling studies of III-group binary nitrides (GaN, AlN) MOVPE in AIX-series reactors were performed ( [2], [9], [10] ).

In this paper the model of group III-nitrides growth by MOVPE is applied to simulation of InGaN growth 
from triethylgallium, trimethylindium and ammonia in AIX 200 Reactor and AIX 2000 HT Planetary Reactor. The results are compared to the data of special experimental studies.

\section{Modeling study of $\ln _{x} \mathrm{Ga}_{1-\mathrm{x}} \mathrm{N}$ growth by MOVPE}

In order to perform the detailed analysis of processes taking place during the growth the we use the model based on the 2D steady-state Navier-Stokes equations of fluid dynamics with taking into account all important physical processes such as conjugated heat transfer, radiation, multicomponent diffusion as well as gasphase chemical reactions. The details of solution procedure are described elsewhere [11], [12].

Gas phase chemical reaction mechanism involves pyrolysis of metal-organic indium and gallium precursors. Kinetics of trimethylindium decomposition was studied in paper [13] where two-step mechanism based on elimination of methyl radicals was suggested

$$
\begin{aligned}
& \text { TMIn } \rightarrow \text { MMIn }+2 \mathrm{CH}_{3} \quad \mathrm{k}=5.0119 \cdot 10^{15} \cdot \exp (-23753.3 / \mathrm{T}) \\
& \text { MMIn } \rightarrow \text { In }+\mathrm{CH}_{3} \quad \mathrm{k}=8.1283 \cdot 10^{10} \cdot \exp (-19488.7 / \mathrm{T})
\end{aligned}
$$

Decomposition of triethylgallium was reported in [14]. Loss of the first ethyl group was found to be the main step of this reaction

$$
\mathrm{TEGa} \rightarrow \mathrm{DEGa}+\mathrm{C}_{2} \mathrm{H}_{5} \quad \mathrm{k}=5.0119 \cdot 10^{15} \cdot \exp (-24105.5 / \mathrm{T})
$$

The possibility of adduct formation due to pre-reaction between group-III metalorganic precursors and ammonia is discussed in several papers [15], [16]. This reaction leading to depletion of group-III precursors is generally assumed to be important during MOVPE of $\mathrm{AlN}$ and $\mathrm{AlGaN}$ but its relative contribution into the $\mathrm{GaN}$ and InGaN growth process is not clearly understood. Moreover there is lack of reliable data on adduct formation kinetics as well as on the nature of adductderived species. That is why we do not include adduct formation into our model. In general this reaction should not be neglected but at the present stage of our study we neglect it for these reasons.

Recent investigations reveal the specific character of chemical processes on group III-nitride surfaces during MOVPE growth - an extremely low sticking probability for molecular nitrogen, non-equilibrium decomposition of ammonia and a significant effect of various growth parameters on the indium incorporation into InGaN layer. An advanced approach to simulation of surface processes during MOVPE of group III-nitrides has been proposed in reference [17]. The model accounts for specific kinetics of nitrogen and ammonia chemical interactions with the growth surface of nitrides by introducing the sticking coefficients of molecular nitrogen and ammonia extracted from independent experimental data. The sticking coefficients of the other species are taken to be unity. Desorption of the volatile species such as indium and gallium is considered in a quasi-equilibrium approximation, allowing one to calculate the desorption rates on the basis of thermodynamic properties of gaseous and solid compounds [18]. This model used in this study does not use any fitting of parameters.

\subsection{Modeling of InGaN growth in AIX 200 Reactor}

The AIX-200 Reactor is an MOVPE system which allows one to perform growth studies in a wide range of operating conditions. It also has a relatively simple design and geometry making its modeling description easier. Taking into account these features we apply modeling to the analysis of the main factors influencing indium incorporation in the InGaN epitaxial layers grown by MOVPE in the commercial AIX-200 horizontal tube reactor and compare the results with the data of experimental measurements.

The experimental study of InGaN growth was performed using triethylgallium (TEGa), trimethylindium (TMIn) and ammonia $\left(\mathrm{NH}_{3}\right)$ as the gallium, indium and nitrogen precursors, respectively. A few series of growth runs were performed to examine the influence of the main growth parameters on the indium incorporation into the epitaxial layer. The total gas flow rate was kept at $4.5 \mathrm{slm}$. The reactor pressure was varied between 50 and $200 \mathrm{mbar}$, the V/III ratio was varied from 3000 to 30000, and the TMIn / (TMIn + TEGa) ratio in the gas phase was changed from 0.2 to 0.8 . Nitrogen was used as a carrier gas. The indium content in the grown InGaN was estimated from the experimentally measured splitting of (0002) lattice reflections in $\theta-2 \theta$ XRD scan.

The indium composition in the solid phase as a function of the gas phase composition $X_{g}=$ TMIn / (TMIn + TEGa) is shown in Figure 2. It can be seen that the discrepancy between theoretical and experimental values rises along with indium gas phase composition and becomes significant at $X_{g}>20 \%$. Usually the deviation of the indium content in the epitaxial layer from the gas phase composition $X_{g}$ is explained by enhanced In desorption from the InGaN surface. However, desorption of In is included into the present model and its effect is not so pronounced as to explain how little In incorporates under the considered conditions. The comparison of measured and calculated values in a wide temperature range shows that this disagreement is systematic and is not related with the choice of growth temperature ( Figure 3).

The operating pressure is the parameter which influences gas flow dynamics in the reactor. Increasing pressure results in a dominant role for natural convection (buoyancy driven flow) which may disturb the species distributions and therefore change the indium incorporation efficiency. But in the considered regimes both theoretical and experimental results reveal very weak 
sensivity of the In composition in $\mathrm{InGaN}$ to the reactor pressure variations (Figure 4). Moreover the measured values of indium composition are remarkably lower than the calculated ones in a whole range of the pressures (if $X_{g}$ is sufficiently high).

Another phenomena which can influence the indium incorporation mechanisms is the frequently observed formation of In droplets on the surface [5], [19]. Keller et al [5] found that the appearance of the droplets is strongly affected by $\mathrm{NH}_{3} / \mathrm{TMIn}$ ratio and growth temperature, and droplets were expected to appear at the surface for high indium content in the gas phase. More detailed experimental studies are necessary to clarify whether the In droplet formation is important for our experimental conditions.

In any case we can conclude that the presently available model is reasonably accurate for the growth processes with In-content in the gas phase less than $20 \%$.

\subsection{Modeling of InGaN growth in the AIX 2000 HT Planetary Reactor}

The multiwafer AIX 2000 HT Planetary Reactor (Figure 5) is a powerful system which is intended for industrial mass production. Therefore, the main requirements for this type of reactor are high reproducibility and good uniformity of grown layers. To satisfy these requirements it is necessary first of all to establish stable laminar gas flow, and avoid the convective instabilities and recirculations leading to precursor losses. Temperature distribution over the growth zone near the susceptor should be uniform since thermal diffusion may play an important role in deposition process and the difference in temperature gradients can be an origin of nonuniformity of indium content and thickness of the grown layers.

The process conditions are chosen to be typical for InGaN growth - the growth temperature is varied from 700 to $850{ }^{\circ} \mathrm{C}, \mathrm{N}_{2}$ is used as a carrier gas. TMIn, TEGa and ammonia are employed as the group III and group V precursors, respectively. Using the numerical solution we obtain the flow pattern (Figure 6), temperature (Figure 7) and species concentration distributions (Figure 8 and Figure 9) as well as the indium content in the grown InGaN layer (Figure 10).

The calculations show that the flow regime is laminar and no convective instabilities or recirculations in the reactor are observed (See Figure 6). Figure 7 indicates that the temperature gradient is highly uniform over the growth zone.

Gas-phase transport simulations reveal the fact that TMIn decomposes completely due to relatively high temperatures in the vicinity of the wafer (see Figure 8) and that the growth is provided mainly by In-vapor reaching the substrate (Figure 9).
The results of modeling the indium incorporation into a InGaN layer are shown in Figure 10. One can see that the model predictions confirm the high uniformity of indium composition along the substrate radius obtained in the experiments. The calculated values of In composition are in a reasonable agreement with the experimental data. The predicted variation of indium composition over the substrate does not exceed $1 \%$.

The results of modeling are confirmed by experimental observation that highly inform epitaxial layers of $\mathrm{GaN}$ and InGaN can be grown in this reactor under optimized conditions [20]. A typical PL mapping of a InGaN layer grown in the Planetary Reactor is shown in Figure 11. The average wavelength of photoluminescence peak is $414.41 \mathrm{~nm}$ with a standard deviation of $1.65 \mathrm{~nm}$.

\section{Conclusions}

The results of modeling analysis of $\mathrm{InGaN}$ growth by MOVPE are reported in this paper. The proposed growth model is applied to the analysis of the basic factors influencing In incorporation into InGaN layers in an AIX-200 horizontal reactor and results are compared to the data of special experimental studies. There was shown that the model allowed to describe the growth processes with $X_{g}=$ TMIn / (TMIn+TEGa ) $<20 \%$ with a reasonable agreement with the experimental data without any fitting procedure. At higher TMIn/ (TMIn+TEGa ) disagreement between theory and experiment is observed. These disagreement becomes significant at $X_{g}$ higher than $20 \%$. This result shows that there are some physical mechanisms limiting indium incorporation in InGaN at high $X_{g}$ not accounted for in the model. The study of such mechanisms is the topic of forthcoming investigations.

The detailed analysis of transport processes during InGaN growth by MOVPE in AIX 2000 HT Planetary Reactor is performed. There was found that the application of the Planetary Reactor to InGaN deposition allowed achievement of high uniformity of the epitaxial layers. The predicted values of indium composition show reasonable agreement with the experimental data.

\section{REFERENCES}

[1] Shuji Nakamura, Gerhard Fasol, The Blue Laser Diode - GaN based Light Emitters and Lasers , (Springer-Verlag, Heidelberg, 1997), .

[2] H. Jurgensen, D. Schmitz, G. Strauch, E. Woelk, M. Dauelsberg, L. Kadinski, Yu. N. Makarov, MRS Internet J. Nitride Semicond. Res. 1, 26 (1996).

[3] D. Schmitz, R. Beccard, O. Schoen, R. Niebuhr, B. Wachtendorf, Holger Juergensen, MRS Internet J. Nitride Semicond. Res. 2, 9 (1997).

[4] T. Matsuoka, N. Yoshimoto, T. Sasaki, A. Katsui, J. Electron. Mater. 21, 157-163 (1992). 
[5] S. Keller, B. Keller, D. Kapolnek, U. Mishra, S. DenBaars, I. Shmagin, R. Kolbas, S. Krishnankutty, J. Cryst. Growth 170, 349-352 (1997).

[6] EL Piner, MK Behbehani, NA El-Masry, FG McIntosh, JC Roberts, KS Boutros, SM Bedair, Appl. Phys. Lett. 70, 461-463 (1997).

[7] A. Sohmer, J. Off, H. Bolay, V. Härle, V. Syganow, Jin Seo Im, V. Wagner, F. Adler, Andreas Hangleiter, A. Dörnen, Ferdinand Scholz, D. Brunner, O. Ambacher, H. Lakner, MRS Internet J. Nitride Semicond. Res. 2, 14 (1997).

[8] E. L. Piner, F. G. McIntosh, J. C. Roberts, K. S. Boutros, M. E. Aumer, V. A. Joshkin, N. A. El-Masry, S. M. Bedair, S. X. Liu, Mater. Res. Soc. Symp. Proc. 449, 85 (1997).

[9] M Dauelsberg, L Kandinski, YuN Makarov, E Woelk, G Strauch, D Schmitz, H Jurgensen, Inst. Phys. Conf. Ser. 142, 887 (1996).

[10] A.O. Galjukov, Y.E. Egorov, Y.N. Makarov, R.A. Talalaev, C. Kirchner, M. Kamp, K.J. Ebeling, in , Edited by: , C.R. Abernathy, M. Kamp, W.D. Brown, T.D. Moustakas, D.N. Buckley, S.J. Pearton, J.P. Dismukes, F. Ren, (The Electrochemical Society, Inc., Pennington, 1997) 244-252.

[11] Yu.E. Egorov, Yu.N. Makarov, A.O. Galjukov, Yu.B. Magidina, D.Kh. Ofengeim, M.S. Ramm, R.A. Talalaev, A.I. Zhmakin, A.N. Vorob'ev, "Computation of chemically reacting flows in epitaxial reactors using 2D and 3D unstructured grids", in Computational Fluid Dynamics '98, edited by Kyriacos D. Papailiou, Demos Tsahalis, Jacques Periaux, Charles Hirsh, Maurizio Pandolfi, published by John Wiley \& Sons, Ltd.(Chichester), 1008 (1998)

[12] Yu. E. Egorov, A. I. Zhmakin, Comp. Mater. Sci. 11, 204 (1998).

[13] M. P. Jacko, S. J. W. Price, Can. J. Chem. 42, 1198 (1964).

[14] M. C. Paputa, S. J. W. Price, Can. J. Chem. 57, 3178 (1979).

[15] C. H. Chen, H. Liu, D. Steigerwald, W. Imler, C. P. Kuo, M. G. Craford, M. Ludowise, S. Lester, J. Amano, J. Electron. Mater. 25, 1004 (1996).

[16] A. Thon, T. F. Kuech , Appl. Phys. Lett. 69, 55-57 (1996).

[17] S. Yu. Karpov, V. G. Prokofyev, E. V. Yakovlev, R. A. Talalaev, Yu. N. Makarov, MRS Internet J. Nitride Semicond. Res. 4, 4 (1999).

[18] I. N. Przhevalskii, S. Yu. Karpov, Yu. N. Makarov, MRS Internet J. Nitride Semicond. Res. 3, 30 (1998).

[19] Masaya Shimizu, Kazumasa Hiramatsu, Nobuhiko Sawaki, J. Cryst. Growth 145, 209 (1994).

[20] R. Beccard, O. Schon, B. Schineller, D. Schmitz, M. Heuken, H. Juergensen, Mater. Res. Soc. Symp. Proc. 482, 155 (1998).

\section{FIGURES}

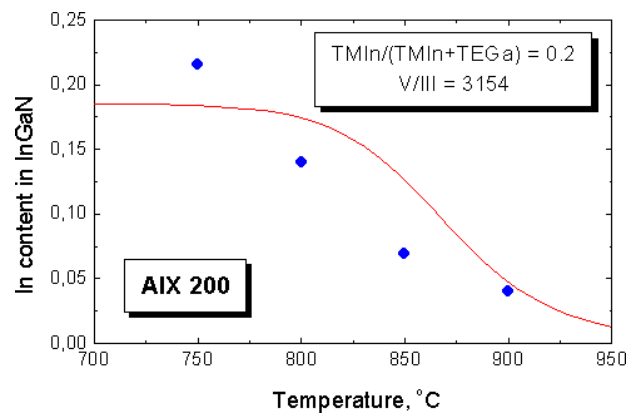

Figure 1. Solid phase composition of InGaN layer grown in AIX-200 horizontal reactor as a function of substrate temperature at low $\mathrm{In} /(\mathrm{In}+\mathrm{Ga})$ ratio in the gas phase. The reactor pressure is 50 mbar. Solid line represents results of the calculations, circles are the experimental data.

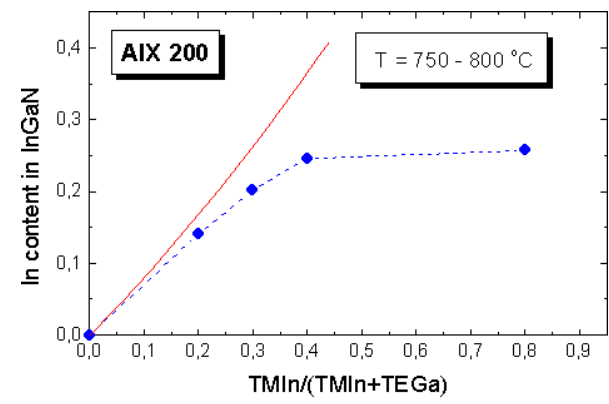

Figure 2. Indium content in InGaN grown in AIX-200 horizontal reactor as a function of TMIn/(TMIn+TEGa) gas phase ratio. The reactor pressure is $50 \mathrm{mbar}$. Solid line presents modeling results, circles are the experimental data.

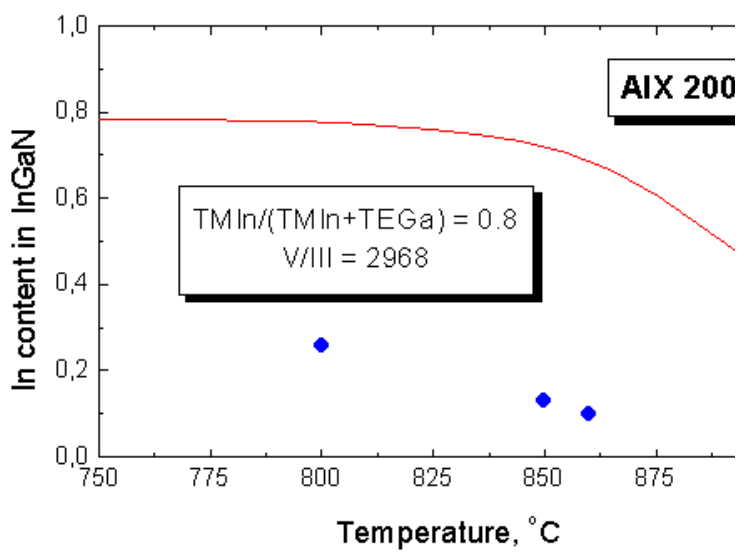

Figure 3. Indium content in InGaN grown in AIX-200 horizontal reactor as a function of substrate temperature at high $\mathrm{In} /(\mathrm{In}+\mathrm{Ga})$ gas phase ratio. The reactor pressure is 50 mbar. Solid line presents the modeling results, circles are the experimental data. 


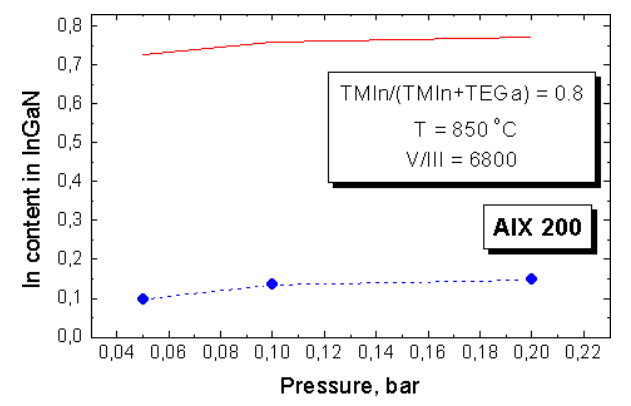

Figure 4. Indium content in InGaN grown in AIX-200 horizontal reactor as a function of reactor pressure. Red line presents the modeling results, circles - experimental data.

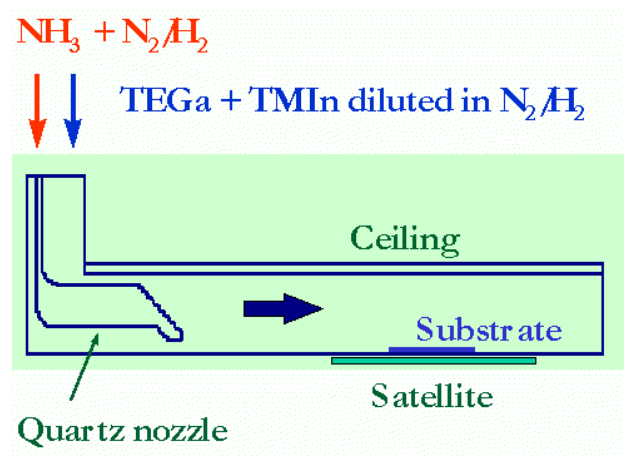

Figure 5. Scheme of AIX 2000 HT Planetary Reactor.

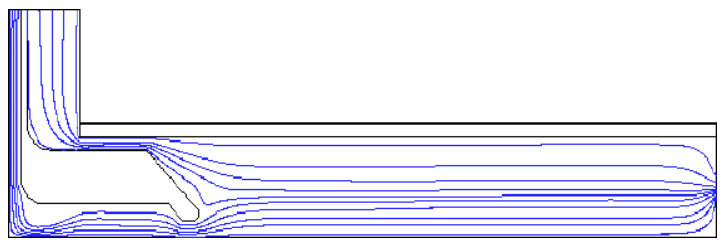

Figure 6. Modeling analysis of InGaN growth by MOVPE in AIX 2000 HT Planetary Reactor - flow pattern

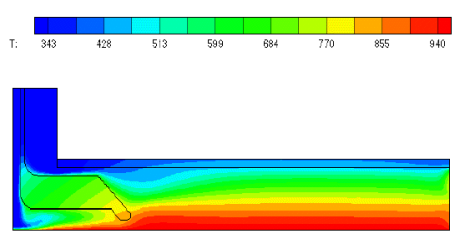

Figure 7. Modeling analysis of InGaN growth by MOVPE in AIX 2000 HT Planetary Reactor - temperature contours.

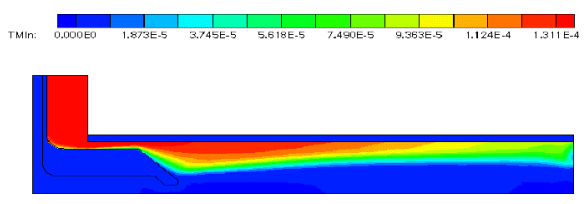

Figure 8 . Modeling analysis of InGaN growth by MOVPE in AIX 2000 HT Planetary Reactor - TMIn mass fraction contours.

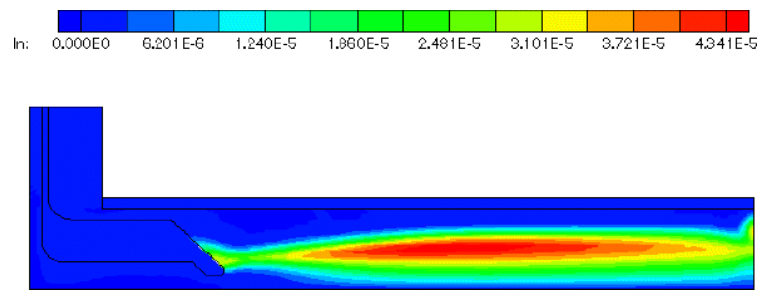

Figure 9. Modeling analysis of InGaN growth by MOVPE in AIX 2000 HT Planetary Reactor - In mass fraction contours.

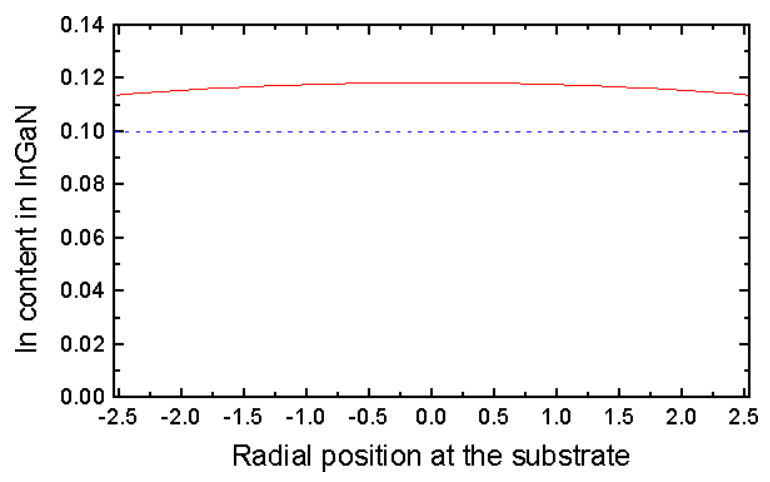

Figure 10. Modeling analysis of InGaN growth by MOVPE in AIX 2000 HT Planetary Reactor - indium content in InGaN as the function of radial position at the substrate. Solid line presents the modeling results, dashed line is the average experimental value.

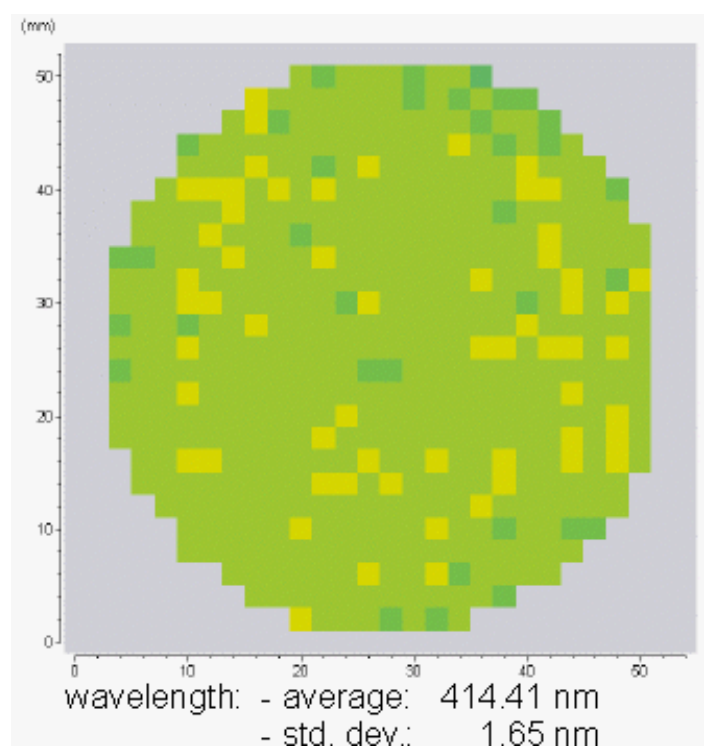

Figure 11. PL mapping of InGaN layer grown in the Planetary Reactor. 